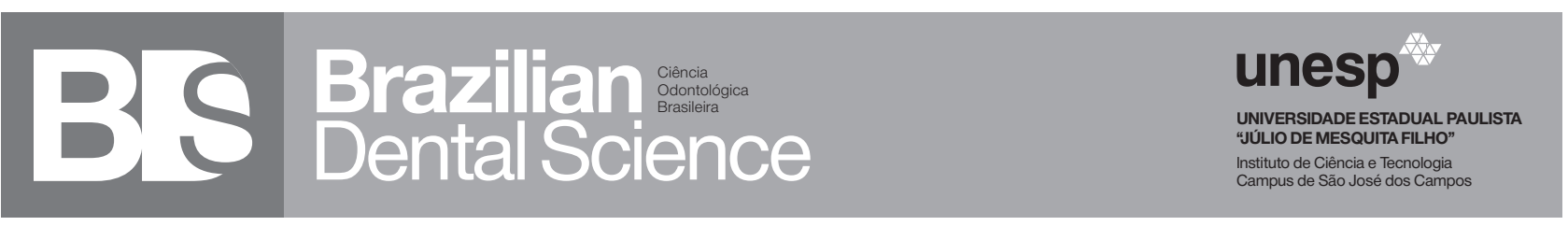

\title{
CHINA: A COUNTRY TO BE DISCOVERED
}

In recent years, I have been contemplating the possibility of building professional bridges between Brazil and Asia. In Oral Radiology, which is my area of expertise, this continent stands out for presenting important technological development and a research society with qualified intellectual production. In addition, although the Postgraduate Program in Oral Radiology of the Piracicaba Dental School within the University of Campinas (FOP/UNICAMP) has presented relevant history of internationalization with countries in Europe, North America and Oceania, our collaboration coverage should also focus on other geographical directions.

In the second half of 2017, the executive board of international relations at UNICAMP opened a call for proposals to encourage coordinators of postgraduate programs to carry out technical visits around the world in order to network with universities of the Santander Bank, which is the funder. This was a great opportunity for me to put into practice an embryonic plan. Therefore, I contacted the head of the Oral Radiology section at Peking University, in Beijing, who promptly accepted my proposal and gave his best to make this amazing opportunity become a reality. Fortunately, my application was approved and, as a way to optimize the trip, I also contacted the University of Hong Kong as they also have excellent scientific representation in Oral Radiology.

This experience has definitely brought me many positive outcomes, starting with the confirmation of my perception of the pronounced international recognition of
Brazilian research. The infrastructure of both universities I visited is fantastic: new and modern facilities, state-of-the-art equipment for the most diverse areas of Dentistry and an impressive number of treated patients. For instance, the Radiology clinic of Peking University produces around 150 radiographic examinations per day, which is quite a high number. In addition, I also had the opportunity to give a lecture on radiological analysis in clinical research at Peking University and to introduce the postgraduate program I coordinate, as well as FOP/UNICAMP.

Although I was aware that China is a country with the largest population in the world, this characteristic was the most impressive to me on a daily basis: with its overcrowded streets, sidewalks, intersections, subway stations and airports. However, in many places, there is infrastructure to organize and assist urban mobility. Also, I felt extremely safe. In many locations there is policing, camera monitoring, and X-ray detector inspection. These may explain the comfort people have to use electronics and handle money in public. Still, during the 15 days I spent in China, I encountered only two homeless people, which is positively impressive compared to many Brazilian capitals.

I found everything to be very organized. Hong Kong and Shanghai are modern with clear preserved tradition. Beijing is more classical and closer to the picture I had of China as a whole. In Beijing, air pollution is more impactful and this became evident with the large number of people wearing masks on the street, and the dark fog, often times covering 


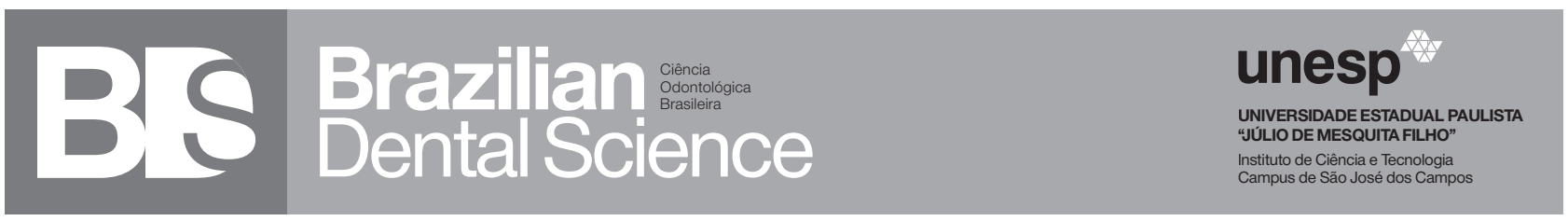

the sun. This air condition, added to the pollen from the beginning of spring, severely irritated my airways.

When studying about China, I found it very interesting to learn about the differences between Hong Kong, Macau and the rest of the country, known as Mainland China. Currently, Hong Kong belongs to China and is part of the Asian tigers, but, for many years, its sovereignty belonged to the United Kingdom. This makes it an independent administrative region with different currency, customs, dialect and censorship laws. In Hong Kong, English communication is very efficient and commonly used. In Shanghai and Beijing, although most of the signs were in two languages, I came across some embarrassing situations for not being able to speak Mandarin.

Since my trip to China was confirmed, I started to meet many Brazilians who claim that they would never go on an adventure like this. However, I emphasize that visiting China can be indeed a very interesting experience, as long as the visitor is open-minded to understanding the beauty of cultural diversity. Even being prepared for many unusual situations, adventures always come with some surprises. I hope that national research funding agencies get restored and that postgraduate students get increasingly encouraged to work in partnership with leading universities from all over the world, reducing favoritism to those in specific countries. Based on my experience, I strongly believe that many of you would start admiring China as a country to be discovered.

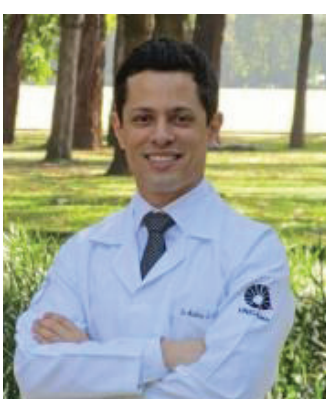

Matheus Lima de Oliveira

University of Campinas (UNICAMP) - School of Dentistry at Piracicaba - Piracicaba - SP Brazil.

E-mail: matheuso@unicamp.br 


\section{CHINA: UIM PAÍS A SER DESCOBERTO}

Há alguns anos, eu venho pensando na possibilidade de iniciar a construção de pontes profissionais entre o Brasil e a Ásia. Em Radiologia Odontológica, que é a minha área de atuação, esse continente se destaca por apresentar importante desenvolvimento tecnológico e uma sociedade de pesquisa com qualificada produção intelectual. Além disso, embora o Programa de Pós-Graduação em Radiologia Odontológica da Faculdade de Odontologia de Piracicaba da Universidade Estadual de Campinas (FOP/UNICAMP) tenha apresentado histórico relevante de internacionalização com países da Europa, América do Norte e Oceania, também se identificava a necessidade de focar a nossa abrangência de colaboração para outras direções geográficas.

No segundo semestre de 2017, a Diretoria Executiva de Relações Internacionais da UNICAMP abriu um edital com a finalidade de incentivar coordenadores de programa de pós-graduação a realizar visitas técnicas a universidades internacionais integrantes da rede de relacionamentos do banco financiador, o Banco Santander. Foi assim que eu vi nesse edital uma grande oportunidade de colocar em prática um planejamento ainda incipiente. Dessa forma, eu fiz contato com o professor responsável pela Radiologia Odontológica da Universidade de Pequim, que atendeu prontamente à minha proposta e se colocou à disposição para a viabilização dessa visita. Felizmente, a minha candidatura junto ao edital foi aprovada e, como forma de otimizar o aproveitamento dessa viagem, também estabeleci contato com a Universidade de Hong Kong, cuja área de Radiologia Odontológica apresenta excelente representação científica.
Muitos foram os frutos positivos dessa experiência, a começar pela confirmação da percepção do grande reconhecimento que a pesquisa brasileira tem no exterior. A infraestrutura de ambas as universidades visitadas é fantástica: instalações novas e modernas, equipamentos de ponta para as mais diversas áreas da Odontologia e números relacionados à quantidade de pacientes atendidos que impressionam. Como um exemplo, a clínica de radiologia da Universidade de Pequim produz aproximadamente 150 exames radiográficos por dia, o que é um número bastante elevado. Ainda, nessa mesma escola, eu tive a oportunidade de dar uma palestra sobre análises radiográficas em pesquisa clínica e apresentar o programa de pós-graduação que eu coordeno, assim como a FOP/UNICAMP.

Embora não fosse novidade para mim de que a China é o país mais populoso do mundo, essa foi uma das características que mais me impressionou no dia-a-dia: ruas, calçadas, cruzamentos, estações de metrô e aeroportos superlotados. No entanto, em muitos locais, há infraestrutura para organizar e auxiliar bastante a mobilidade urbana. Além disso, eu tive boa sensação de segurança. Em muitos ambientes há policiamento, monitoramento por câmeras e inspeção por detectores de raios $\mathrm{X}$. Talvez, esses sejam motivos que justifiquem a tranquilidade com que pessoas fazem uso de eletrônicos e exibem dinheiro pelas ruas. Ainda, durante os 15 dias que passei pela China, deparei-me somente com dois moradores de rua, o que é algo positivamente impressionante, se comparado a muitas capitais brasileiras. 
Eu achei tudo muito organizado. Hong Kong e Xangai são modernas, mas com claros sinais de conservação da tradição. Pequim é clássica e mais próxima da imagem que eu tinha da China como um todo. Em Pequim, a poluição do ar é mais impactante do que nas demais cidades que eu visitei. Isso se torna evidente pelo grande número de pessoas que fazem uso de máscara nas ruas e pelo céu cinzento que, algumas vezes, encobre totalmente o sol. Essa condição do ar, somado ao pólen do início da primavera, causou em mim grande irritação das vias aéreas.

Ao estudar sobre a China, achei muito interessante conhecer as diferenças existentes entre Hong Kong, Macau e o restante do país, conhecido como China Continental. Atualmente, Hong Kong pertence à China e é parte dos tigres asiáticos, mas, por muitos anos, a sua soberania pertenceu ao Reino Unido. Isso faz com que ela seja uma região administrativa independente com moeda, leis aduaneiras, dialetos e censura diferentes. Em Hong Kong, a comunicação em inglês é muito eficiente e bastante utilizada. Já em Xangai e Pequim, embora a maioria das placas de sinalização seja em duas línguas, deparei-me com algumas situações complicadas por eu não falar mandarim.
Desde que a minha viagem a China foi confirmada, comecei a perceber que há brasileiros que jamais se submeteriam a essa aventura. No entanto, ressalto que visitar a China é, realmente, uma experiência muito interessante. É preciso que o visitante esteja aberto à compreensão da riqueza que existe na diversidade cultural. Por mais que possamos nos preparar para situações inusitadas, há sempre alguma experiência nova que nos surpreende. Espero que as agências nacionais de fomento à pesquisa se restabeleçam e que alunos de pós-graduação estejam cada vez mais estimulados a trabalhar em parceria com universidades de destaque do mundo todo, reduzindo o favoritismo àquelas que se situam em países específicos. Baseado na minha experiência, acredito fortemente que muitos de vocês passariam a admirar a China como um país a ser descoberto.

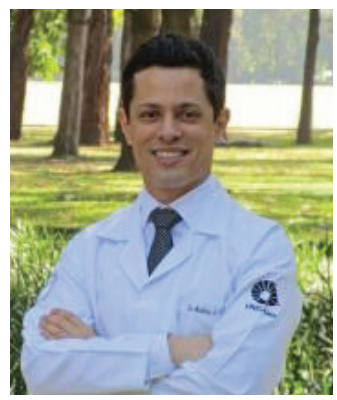

Matheus Lima de Oliveira

Universidade de Campinas - Faculdade de odontologia de Piracicaba - SP - Brasil. E-mail: matheuso@unicamp.br 